\title{
Introducing a Watermarking with a Multi-Objective Genetic Algorithm
}

\author{
Diego Sal Díaz \\ Dpt. CCIA, UPV/EHU \\ Comp. Intelligence Group \\ San Sebastian, Spain
}

\author{
Manuel Graña Romay * \\ Dpt. CCIA, UPV/EUH \\ Comp. Intelligence Group \\ San Sebastian, Spain
}

\begin{abstract}
We propose an evolutionary algorithm for the enhancement of digital semi-fragile watermaking based on the manipulation of the image discrete cosine transform (DCT). The algorithm searches for the optimal localization of the DCT of an image to place the mark image DCT coefficients. The problem is stated as a multi-objective optimization problem (MOP), that involves the simultaneous minimization of distortion and robustness criteria.
\end{abstract}

\section{Categories and Subject Descriptors}

1 [Real World Applications]: Watermarking; 2 [Evolutive Strategies]: Multi-objective Optimization.

\section{General Terms}

Algorithms

\section{Keywords}

Genetic Algorithm, Multi-Objective, Watermarking.

\section{INTRODUCTION}

Semi-fragile watermarking $[7,1,5,6,8]$ tries to ensure the image integrity, by means of an embedded watermark which can be recovered without modification if the image has not been manipulated. If the image suffers some modification, the watermark is corrupted. However, in order to prove ownership, it is interesting that the mark is robust to operations like filtering, smoothing and lossy compression which are very common while distributing images though communication networks. For several reasons (privacy, minimal perturbation of the image content, etc) a watermarked image must be as indistinguishable of the original one as possible. The watermarking process must introduce the minimum possible visual distortion in the image. These two requirements are the objectives of our work and can be contradicting in some instances. Combined optimization of the distortion and the robustness can be stated as a multi-objective optimization.

${ }^{*}$ The Spanish Ministerio de Educacion y Ciencia supports this work through grants DPI2003-06972 and VIMS-200320088-c04-04.

Copyright is held by the author/owner. GECCO'05, June 25-29, 2005, Washington, DC, USA. ACM 1-59593-010-8/05/0006.
In the problem of searching for an optimal placement of the watermark image, the trade-off between robustness and image fidelity is represented by the Pareto-Front $[2,3]$ discovered by the algorithm. We define an evolutive strategy that tries to provide a sample of the Pareto-Front preserving as much as possible the diversity of the solutions.

\section{MULTI-OBJECTIVE OPTIMIZATION PROBLEM}

The general MOP try to find the vector $\vec{x}^{*}=\left[x_{1}^{*}, x_{2}^{*}, \ldots, x_{n}^{*}\right]^{T}$ which will satisfy the $m$ inequality constraints $g_{i}(\vec{x}) \geq 0, i=$ $1,2, \ldots, m$, the $p$ equality constraints $h_{i}(\vec{x})=0, i=1,2, \ldots, p$ and will optimize the vector function $\vec{f}(\vec{x})=\left[f_{1}(\vec{x}), f_{2}(\vec{x}), \ldots\right.$, $\left.f_{k}(\vec{x})\right]^{T}$. A vector $\vec{x}^{*} \in \mathcal{F}$ is Pareto optimal if it does not exist another $\vec{x} \in \mathcal{F}$ such that $f_{i}(\vec{x}) \leq f_{i}\left(\vec{x}^{*}\right)$ for all $i=1, . ., k$ and $f_{j}(\vec{x})<f_{j}\left(\vec{x}^{*}\right)$ for at least one $j$. Each solution that carries this property, is called non-dominated solution, and the set of non-dominated solutions is called Pareto optimal set. The plot of the objective functions whose non-dominated vectors are in the Pareto optimal set is called the Pareto-Front. A vector $\vec{u}=\left(u_{1}, \ldots, u_{n}\right)$ is said to dominate $\vec{v}=\left(v_{1}, \ldots, v_{n}\right)$ (denoted as $\vec{u} \preceq \vec{v}$ ) if and only if $\forall i \in\{1 . . k\}, u_{i} \leq v_{i} \wedge \exists i \in\{1, \ldots, k\}: u_{i}<v_{i}$. For a given MOP $\vec{f}(x)$, the Pareto optimal set $\mathcal{P}^{*}$ is defined as: $\mathcal{P}^{*}:=\left\{x \in \mathcal{F} \mid \neg \exists x^{\prime} \in \mathcal{F}: \vec{f}\left(x^{\prime}\right) \preceq \vec{f}(x)\right\}$, and the Pareto-Front $\left(\mathcal{P} \mathcal{F}^{*}\right)$ is defined as: $\mathcal{P} \mathcal{F}^{*}:=\{\vec{u}=\vec{f}=$ $\left.\left(f_{1}(x), \ldots, f_{k}(x)\right) \mid x \in P^{*}\right\}$.

\section{ALGORITHM}

For obvious space restrictions we will only comment the definition of the vector fitness function composed of the robustness and distortion fitness functions [4].

Robustness fitness function $f_{1}$ : Robustness refers to the property of recovering the mark even when the watermarked image has been manipulated. We are interested in having robustness against lossy compression and smoothing. Both transformations affect the high and preserve the low frequency transform coefficients. Therefore the closer to the origin of the transform space the mark is located, the higher the robustness of the mark. As we are embedding the logo DCT, we note that most of the information will be in the low frequency coefficients so, they must have priority to be embedded in the positions that are nearer to the low frequencies of the image DCT.

Distortion fitness function $f_{2}$ : The true distortion is computed as the squared difference between the original im- 


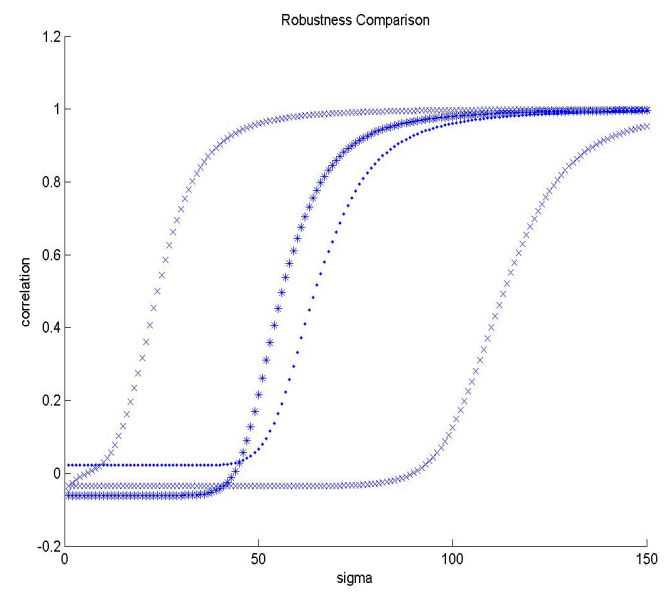

Figure 1: Robustness comparison by means of the correlation coefficient of the recovered image mark versus the radius of the smoothing convolution kernel.

age and the inverse of the marked DCT. Final results in the figures will reflect this value. However, to avoid the computational cost of the DCT inversion, we use as the fitness function of the evolutionary algorithm an approximation that follows from the observation that the distortion introduced adding something to a DCT coefficient is proportional to the absolute value of that coefficient.

\section{RESULTS}

Figure 1 plots the correlation coefficient between the original watermark and the corrupted watermark recovered after the marked image has been smoothed by a low-pass gaussian filter with increasing filter radius sigma applied in the Fourier transform domain. Solutions with lowest distortion and robustness in the Pareto-From are represented by ' $x$ '. The solution with best robustness under a given distortion taken from de GA Pareto-Front is represented by '*'. The ' ' represent the same taken from the local search algorithm. That means that the GA found a solution that is much more robust than the distortion optimal one while preserving much of the distortion quality. Figure 3 shows the visual results of the recuperation of the mark image after smoothing the image watermarked using the placement from the MOP-GA solution.

The second class of attacks we are considering are the lossy compression. We apply the jpeg compression with increasing quality factor to the image after the watermark, and we recover the watermark image from the decompressed image. Figure 2 shows the correlation of the recovered mark relative to the true mark versus compression quality, for both the local search and GA solutions. It can be appreciated that the GA solution recovers much better than the local search solution from strong lossy compression.

\section{REFERENCES}

[1] D. Augot, J. Boucqueau, J. Delaigle, C. Fontaine, and E. Goray. Secure delivery of images over open networks. Proceedings of the IEEE, 87(7):1251-1266, July 1999.

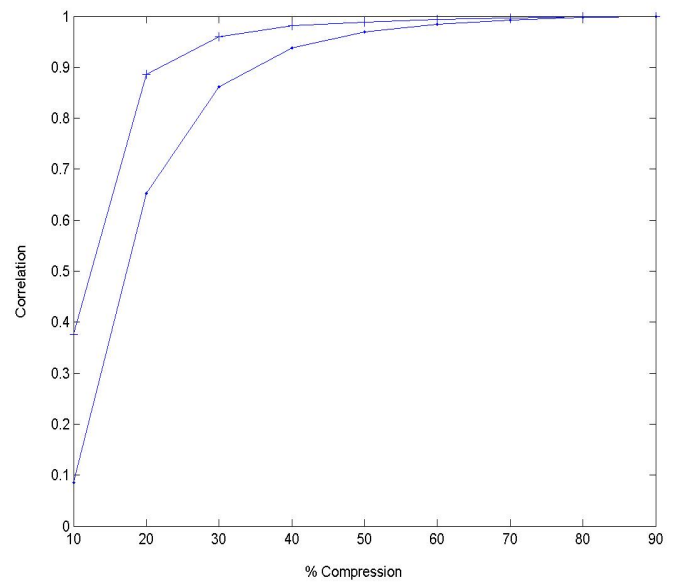

Figure 2: Robustness of solutions from GA '? and Local Search ' + ' to JPEG compression. Correlation of the recovered mark image versus compression quality

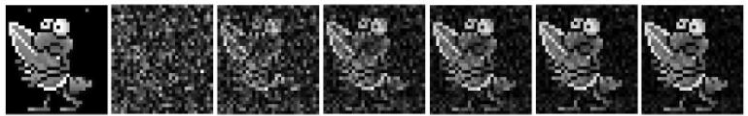

Figure 3: Watermark logo: Original and recovered from GA solution after low-pass filtering the watermarked image with $\sigma=50,60,70,80,90,100$.

[2] C. Coello Coello, G. Toscano Pulido, and

E. Mezura Montes. Current and future research trends in evolutionary multiobjective optimization. In M. Graña, R. Duro, A. d'Anjou, and P. Wang, editors, Information Processing with Evolutionary Algorithms, pages 213-232. Springer Verlag, New York, 2004.

[3] K. Deb, A. Pratap, S. Agarwal, and T. Meyarivan. A fast and elitist muliobjective genetic algorithm: Nsga-ii. IEEE transactions on evolutionary computation, 6(2):182-197, April 2002.

[4] D. Sal and M. Graña. Multi-objective genetic algorithm for semi-fragile watermarking. In JCIS Proceedings, volume in press, 2005.

[5] P. B. Schneck. Persistent access control to prevent piracy of digital information. Proceedings of the IEEE, 87(7):1239-1250, July 1999.

[6] C. Vleeschouwer, J. Delaigle, and M. B. Invisibility and application functionalities on perceptual watermarkingan overview. Proceedings of the IEEE, 90(1):64-77, January 2002.

[7] G. Voyatzis and I. Pitas. The use of watermarks in the protection of digital multimedia products. Proceedings of the IEEE, 87(7):1197-1207, July 1999.

[8] R. B. Wolfgang, C. I. Podilchuk, and E. J. Delp. Perceptual watermarking for digital images and video. Proceedings of the IEEE, 87(7):1108-1126, July 1999. 